\title{
Sentinel Lymph Node Mapping for Endometrial Cancer: A Modern Approach to Surgical Staging
}

\author{
Nadeem R. Abu-Rustum, MD
}

\begin{abstract}
Most patients with endometrial cancer will present with early-stage disease. Although the rate of metastasis in these patients is low, proffering excellent prognoses, the standard of treatment in many practices still includes a complete or selective pelvic and para-aortic lymphadenectomy for staging; and accurate surgical staging is the most important prognostic factor. Many patients will undergo a comprehensive lymphadenectomy despite having disease confined to the uterus, resulting in prolonged operating time, additional cost, and potential side effects, such as lower extremity lymphedema. However, recent studies show that a complete lymphadenectomy may have no therapeutic benefit in patients with early-stage endometrial cancer. Sentinel lymph node (SLN) mapping, which has been used in other cancer types, may be an acceptable surgical strategy between a complete lymphadenectomy and no nodal evaluation in patients with endometrial cancer. SLN mapping is based on the concept that lymph node metastasis is the result of an orderly process; that is, lymph drains in a specific pattern away from the tumor, and therefore, if the SLN, or first node, is negative for metastasis, then the nodes after the SLN should also be negative. This approach can help patients avoid the side effects associated with a complete lymphadenectomy, although disease must be thoroughly staged for accurate prognosis and determination of appropriate treatment approach. Surgeon experience, adherence to an SLN algorithm, and the use of pathologic "ultrastaging" are key factors for successful SLN mapping. (J Natl Compr Canc Netw 2014;12:288-297)
\end{abstract}

\section{NCCN: Continuing Education}

\section{Accreditation Statement}

This activity has been designated to meet the educational needs of physicians and nurses involved in the management of patients with

From the Gynecology Service, Department of Surgery, Memorial Sloan-Kettering Cancer Center, New York, New York.

Submitted July 23, 2013; accepted for publication December 6, 2013.

The author has disclosed that he has no financial interests, arrangements, affiliations, or commercial interests with the manufacturers of any products discussed in this article or their competitors.

Correspondence: Nadeem R. Abu-Rustum, MD, Gynecology Service, Department of Surgery, Memorial Sloan-Kettering Cancer Center, 1275 York Avenue, New York, NY 10065. E-mail: Abu-rusn@mskcc.org

\section{EDITOR}

Kerrin M. Green, MA, Assistant Managing Editor, JNCCN_Journal of the National Comprehensive Cancer Network

Ms. Green has disclosed that she has no relevant financial relationships. cancer. There is no fee for this article. No commercial support was received for this article. The National Comprehensive Cancer Network (NCCN) is accredited by the ACCME to provide continuing medical education for physicians.

NCCN designates this journal-based CME activity for a maximum of 1.0 AMA PRA Category 1 Credit(s) ${ }^{\mathrm{TM}}$. Physicians should claim only the credit commensurate with the extent of their participation in the activity.

NCCN is accredited as a provider of continuing nursing education by the American Nurses Credentialing Center's Commission on Accreditation.

This activity is accredited for 1.0 contact hour. Accreditation as a provider refers to recognition of educational activities only; accredited status does not imply endorsement by NCCN or ANCC of any commercial products discussed/displayed in conjunction with the educational activity. Kristina M. Gregory, RN, MSN, OCN, is our nurse planner for this educational activity.

All clinicians completing this activity will be issued a certificate of participation. To participate in this journal CE activity: 1) review the learning objectives and author disclosures; 2 ) study the education content; 3 ) take the posttest with a $66 \%$ minimum passing score and complete the evaluation at http://education.nccn.org/ node/40436; and 4) view/print certificate.

Release date: February 24, 2014; Expiration date: February 24, 2015

\section{Learning Objectives}

Upon completion of this activity, participants will be able to:

- Describe the importance of lymph node assessment in the treatment of endometrial cancer

- Compare and contrast the surgical strategies of SLN mapping, complete or selective lymphadenectomy, or no nodal evaluation in patients with endometrial cancer

- Define the key factors for successful SLN mapping including adherence to an SLN algorithm and use of "ultrastaging"

\section{CE AUTHORS}

Deborah J. Moonan, RN, BSN, Director, Continuing Education \& Grants Ms. Moonan has disclosed that she has no relevant financial relationships.

Ann Gianola, MA, Manager, Continuing Education \& Grants Ms. Gianola has disclosed that she has no relevant financial relationships.

Kristina M. Gregory, RN, MSN, OCN, Vice President, Clinical Information Operations

Ms. Gregory has disclosed that she has no relevant financial relationships. 
Sentinel lymph node (SLN) mapping is an imageguided procedure that is well established in the treatment of cancers, such as melanoma ${ }^{1}$ and breast cancer. ${ }^{2}$ This approach is based on the concept that lymph drains in an orderly pattern away from the tumor through the lymphatic system; therefore, if the SLN, or first node, is negative for metastasis, then the ensuing nodes should also be negative. A recent study showed that SLNs are 3 times more likely than non-SLNs to harbor metastatic disease. ${ }^{3}$ Gould et $\mathrm{al}^{4}$ coined the term sentinel node in 1960 with his observations of carcinoma of the parotid gland. He suggested that examining the SLN for metastatic disease could help determine whether a patient needed a radical neck dissection. His approach was based on simply identifying the anatomic location of the SLN. In 1977, Cabanas ${ }^{5}$ described the SLN in patients with penile carcinoma, this time with the use of the imaging procedure lymphography. SLN mapping in endometrial cancer was introduced by Burke et $\mathrm{al}^{6}$ in 1996, but has only recently gained popularity. The appeal of SLN mapping lies in the possible avoidance of "overstaging" via lymph node dissection of normal/negative nodes and enhanced precision in finding micrometastasis with pathologic ultrastaging of SLNs. Comprehensive lymphadenectomy may be associated with detrimental side effects, such as lower extremity lymphedema and lymphocysts, which can negatively impact quality of life. It is also likely associated with greater operating time, prolonged anesthesia, increased blood loss, vascular and nerve injury, and an increased conversion rate from laparoscopy to laparotomy to complete the operation successfully. However, because accurate surgical staging is the most important prognostic factor, the standard in many practices continues to include a comprehensive lymphadenectomy. Furthermore, no prospective randomized trials have yet assessed the efficacy of SLN mapping procedures in endometrial cancer, and no long-term survival data are currently available.

\section{The Importance of Lymph Node Assessment}

Endometrial cancer is the most common gynecologic malignancy; an estimated 44,560 women will be diagnosed with the disease in the United States in
2013. ${ }^{7}$ Most of these women will present with earlystage disease (90\%), without metastasis, ${ }^{8}$ limiting the amount of deaths to approximately 8190 per year. ${ }^{7}$ Because most of these tumors will be confined to the uterus (International Federation of Gynecology and Obstetrics [FIGO] stage I), the 5-year overall survival rate in this patient population is $80 \%$ to $90 \% .{ }^{9,10}$ Approximately $10 \%$ to $15 \%$ of these patients will, in fact, have metastatic nodal disease, and nearly $15 \%$ will be deemed to have grade 1 tumors preoperatively on office biopsy or dilatation and curettage will actually have higher-grade disease on final pathologic review after hysterectomy ${ }^{11}$; therefore, it is of utmost importance to stage and treat these patients properly and avoid missing undetected metastatic disease that may upstage the patient.

Most patients with endometrial cancer will undergo initial surgical treatment that will include a total hysterectomy, bilateral salpingo-oophorectomy, and pelvic washings. Although the rate of metastasis in this patient population is low, the standard of treatment also includes a complete or selective pelvic and para-aortic lymphadenectomy for staging disease. Proper surgical staging, the most important prognostic factor, ${ }^{12,13}$ provides information on the actual extent of disease rather than on perceived risks based on uterine factors, such as grade, histology, and depth of myometrial invasion, which helps tailor adjuvant therapy. ${ }^{14}$

Unfortunately, many patients with early-stage endometrial cancer will undergo surgery with insufficient nodal evaluation, with their nodes being palpated, biopsied only if enlarged, or completely ignored..$^{14}$ Studies have shown rates of nodal assessment as low as $30 \%,{ }^{15}$ although that percentage has increased as the importance of nodal assessment has been realized. When surgical staging is inadequately performed, patients can be subjected to unnecessary adjuvant therapy, such as pelvic radiation therapy and its associated side effects. ${ }^{14}$ Based on the current standard of treatment, surgeons are faced with the dilemma of "understaging" versus "overtreating." Traditionally, it has been suggested that the more nodes removed, the better the chance of detecting metastasis, but also the more likely the patient will develop side effects. The use of SLN mapping in patients with endometrial cancer may be an acceptable solution, providing a middle ground between the polarized schools of thought: complete lymph- 
adenectomy and no nodal evaluation. The logic of the SLN approach lies in targeting the "correct" nodes, or those most likely to harbor disease based on lymphatic flow, rather than removing a greater number of nodes to perform thorough staging. Ultimately, the end goal of both approaches is adequate staging. A potential pitfall to SLN mapping is that some, but possibly not all, positive lymph nodes may be removed, leaving the question: does every microscopically positive node need to be removed to stage patients? And is there a potential therapeutic role of removing normal-appearing lymph nodes?

\section{The Road to SLN Mapping in Endometrial Cancer}

In current practice, the importance of lymph node assessment for proper surgical staging in this patient population cannot be stressed enough, although lymph node removal may increase operative time and blood loss, and is associated with vascular injury, lower extremity lymphedema, and lymphocyst formation. ${ }^{8}$ In a study of 1289 patients with uterine corpus malignancies at Memorial Sloan-Kettering Cancer Center, 16 (3.4\%) of 469 patients who had 10 or more lymph nodes removed at surgery developed new postoperative symptomatic leg lymphedema. ${ }^{16}$ Furthermore, 2 large recently reported trials have questioned the survival benefit of a comprehensive lymphadenectomy in this disease. One such study, A Study in the Treatment of Endometrial Cancer (ASTEC), was a randomized multicenter study of more than 1400 patients, which showed no therapeutic benefit to lymphadenectomy in early-stage endometrial cancer ${ }^{17}$; however, almost half of the patients randomized to the lymph node dissection arm had 9 or fewer nodes removed (ie, possible inadequate staging via a non-SLN approach). The ASTEC trial was also criticized because many patients were treated with postoperative radiation regardless of the status of their lymph nodes. In a randomized trial by Panici et $\mathrm{l}^{18}$ that required a minimum of 20 lymph nodes removed per patient, no difference was seen in overall survival. The findings of these studies, however, contradicted earlier retrospective studies, such as that of Kilgore et $\mathrm{al}^{19}$ in 1995 , which reported a significant survival advantage for patients with endometrial adeno- carcinoma undergoing multiple-site pelvic lymph node sampling versus no node sampling, suggesting a therapeutic benefit to lymphadenectomy. More recently, the retrospective study on Survival Effect of Para-Aortic Lymphadenectomy (SEPAL) in endometrial cancer showed that overall survival was significantly improved in select intermediate- and high-risk patients undergoing pelvic and para-aortic lymph node dissection. ${ }^{20}$ However, the median number of nodes removed was much larger than in most studies, and it was also difficult to determine whether the improvement in overall survival was a result of the removal of the para-aortic nodes or of the adjuvant chemotherapy. ${ }^{20}$

In a pilot study of 42 patients with grade 1 endometrioid endometrial cancer, the most common anatomic sites where SLNs were identified were the internal iliac, $52(36 \%)$; external iliac, 43 (30\%); obturator, 34 (23\%); and common iliac regions, $11(8 \%)$; these are the areas involved in a pelvic nodal dissection (Figures 1 and 2). Only 5 cases (3\%) had para-aortic SLN involvement. ${ }^{11}$ The para-aortic nodal dissection may be an area of possible overstaging, which would be limited with successful negative bilateral pelvic SLN map-

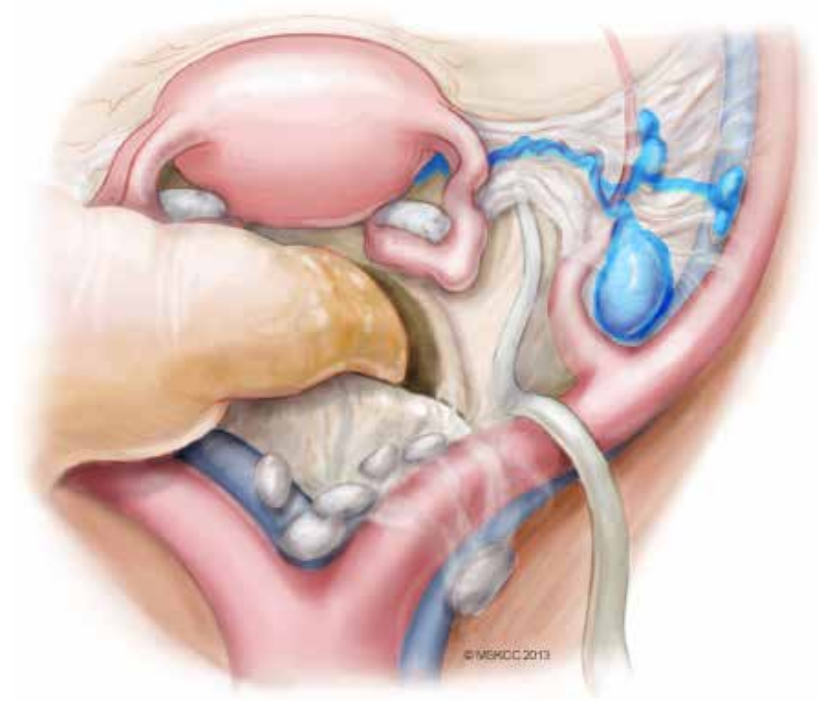

Figure 1 The most common drainage routes, usually when the lymphatic trunks cross over the obliterated umbilical ligament. The most common locations of sentinel lymph nodes after a cervical injection are medial to the external iliac, ventral to the hypogastric, or in the superior part of the obturator space.

Courtesy of Abu-Rustum NR, Levine DA, Barakat RR, eds. Atlas of Procedures in Gynecologic Oncology, 3rd ed. London: Informa Healthcare; 2013. @2013, Memorial Sloan-Kettering Cancer Center. 


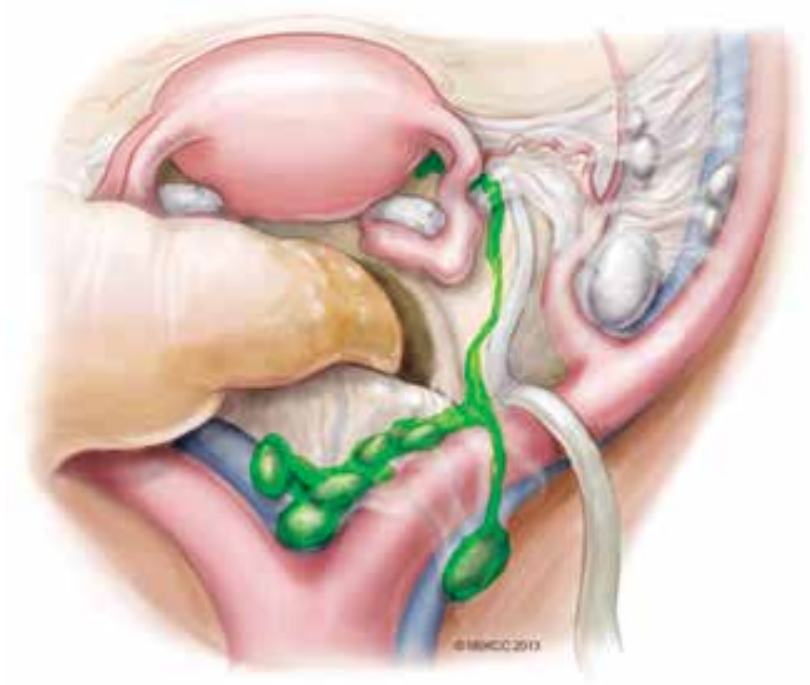

Figure 2 The less common locations of sentinel lymph nodes, usually seen when lymphatic trunks do not cross over the umbilical ligament but follow the mesoureter cephalad to the common iliac and presacral sentinel lymph nodes.

Courtesy of Abu-Rustum NR, Levine DA, Barakat RR, eds. Atlas of Procedures in Gynecologic Oncology, 3rd ed. London: Informa Healthcare; 2013. @2013, Memorial Sloan-Kettering Cancer Center.

ping. In a 2009 study, 847 (44\%) of 1942 patients had both pelvic and para-aortic nodes removed during initial surgery. Only $12(1.6 \%)$ of 734 patients who had negative pelvic nodes had isolated positive para-aortic nodes. When 8 or more negative pelvic nodes were removed, which is a number some consider adequate for accurate pelvic node dissection and staging, only 7 of 640 patients (1\%) had isolated para-aortic nodal metastasis. ${ }^{21}$ In a study of 1920 patients (all had $\geq 1$ node removed) using Classification and Regression Tree (CART) analysis, Barlin et $\mathrm{al}^{13}$ reported no association with the para-aortic nodal assessment and overall survival $(P=.450)$. They also showed that stage I versus stages II-IV and grades 1-2 versus grade 3 (a binary grading system of low-vs high-grade) were predictors of overall survival. In other words, what seems to be important as far as staging is the proper determination of uterine fundus-contained disease versus disease outside the uterine fundus, and the pathologist determining the grade of the tumor as low- versus high-grade (grade 1-2 endometrioid is considered low-grade, and grade 3 endometrioid or serous, clear cell, or carcinosarcoma is considered high-grade).

Even pelvic lymph nodes, which are more likely to contain metastatic disease, may sometimes be un- necessarily removed (overstaged). For example, the "circumflex iliac" lymph nodes are often removed during a routine bilateral pelvic lymphadenectomy, and these nodes are often benign, especially when other nodal areas are also negative. ${ }^{22}$ Removing these nodes may cause lymphatic obstruction to the lower extremity, lower abdominal wall, and pubic region, increasing the risk of lymphedema. ${ }^{23}$

\section{SLN Mapping Techniques}

A radioactive tracer and colored dye (often blue or green) are used to locate "hot" nodes or visualize colored nodes. SLNs are considered positive if they contain macrometastasis (tumor clusters $>2 \mathrm{~mm}$ ), micrometastasis (tumor clusters 0.2-2.0 $\mathrm{mm}$ ), or isolated tumor cells (single tumor cells or tumor clusters $\leq 0.2 \mathrm{~mm}$ ). The treatment of women with only isolated tumor cells in SLNs is still a subject of much current research. SLNs containing only isolated cytokeratin-positive "cells" should be considered negative for metastasis. ${ }^{16}$

Three different types of SLN mapping techniques exist based on site of injection: 1) uterine subserosal, 2) cervical (Figure 3), and 3) endometrial via hysteroscopy. ${ }^{24,25}$ The author's group prefers a cervical injection, although others have argued that a peritumoral injection, either hysteroscopic or fundal, is more appropriate. Their rationale for using a cervical injection includes the following: 1) the main lymphatic drainage to the uterus is from the parametria; therefore, a combined superficial (1-3 mm) and deep (1-2 $\mathrm{cm}$ ) cervical injection is adequate; 2) the cervix is easily accessible; 3) the cervix in women with endometrial cancer is rarely distorted by anatomic variations, such as myomas, which sometimes
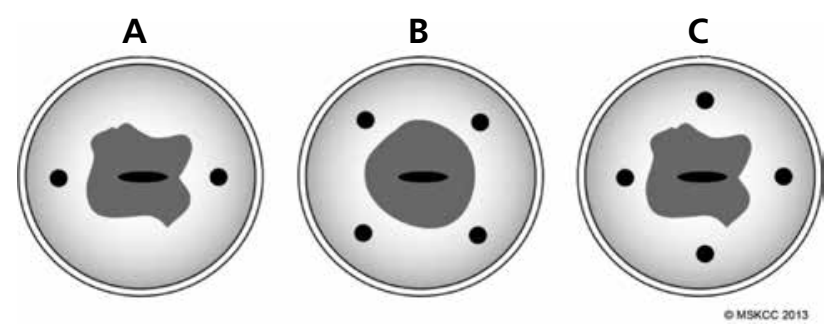

Figure 3 Three different options for direct cervical injection: a 2-sided option ( $A$ ) and the 4-quadrant options (B and C).

Courtesy of Abu-Rustum NR, Levine DA, Barakat RR, eds. Atlas of Procedures in Gynecologic Oncology, 3rd ed. London: Informa Healthcare; 2013. @2013, Memorial Sloan-Kettering Cancer Center. 
make uterine serosal mapping impossible; 4) the cervix in women with endometrial cancer is rarely scarred from prior procedures, such as conization or bulky tumor infiltration; and 5) a uterine fundal serosa mapping does not reflect the parametrial lymphatic drainage of the uterus (the main route of drainage), and most early-stage endometrial cancers do not have disease infiltrating and ulcerating the uterine fundal serosa. ${ }^{26}$ The main argument against the cervical injection is that it has a lower para-aortic detection rate, as opposed to the hysteroscopic approach; but as previously mentioned, when the pelvic lymph nodes are negative for metastasis, disease is unlikely to be found in the para-aortic nodes, ${ }^{21}$ and to date, no definitive well-documented association exists between para-aortic nodal assessment and improved overall survival. ${ }^{13}$ In a recent large meta-analysis, Kang et $\mathrm{al}^{27}$ reported a decrease in detection rates when the cervical method was not used, although without statistical significance. These investigators also recommended that the "subserosal injection-only" technique be avoided because of decreased sensitivity. For these reasons and based on the authors' experience and that of other investigators, they prefer the cervical-only approach and describe the procedure herein.

\section{Lymphoscintigraphy}

A radiolabeled colloid, usually technetium-99 ( $\left.{ }^{99 \mathrm{~m}} \mathrm{Tc}\right)$, is injected into the cervix the day of or the day before surgery. The ${ }^{99 \mathrm{~m}} \mathrm{Tc}$ is then carried via lymph through the lymphatic capillaries to the SLNs. In the "short" protocol (day of surgery), 0.2 to $1.0 \mathrm{mCi}$ of the ${ }^{99 \mathrm{~m}} \mathrm{Tc}$ is injected. In the "long" protocol (20-24 hours before surgery), an additional 2.0 to $4.0 \mathrm{mCi}$ of colloid is used. A preoperative planar lymphoscintigram is taken 20 to 30 minutes after injection for a short protocol, and immediate "dynamic" images and subsequent "static" images are taken to locate the nodes. Gamma probes, such as a laparoscopic SLN probe or a handheld open-procedure SLN probe, are used to detect the hot nodes. Single-photon emission computed tomography (SPECT), which uses 3-dimensional localization of hot nodes also detected through a gamma probe, can be used. ${ }^{28}$

Colored Dye Injection: The colored dye (isosulfan blue 1 , methylene blue $1 \%$, or patent blue

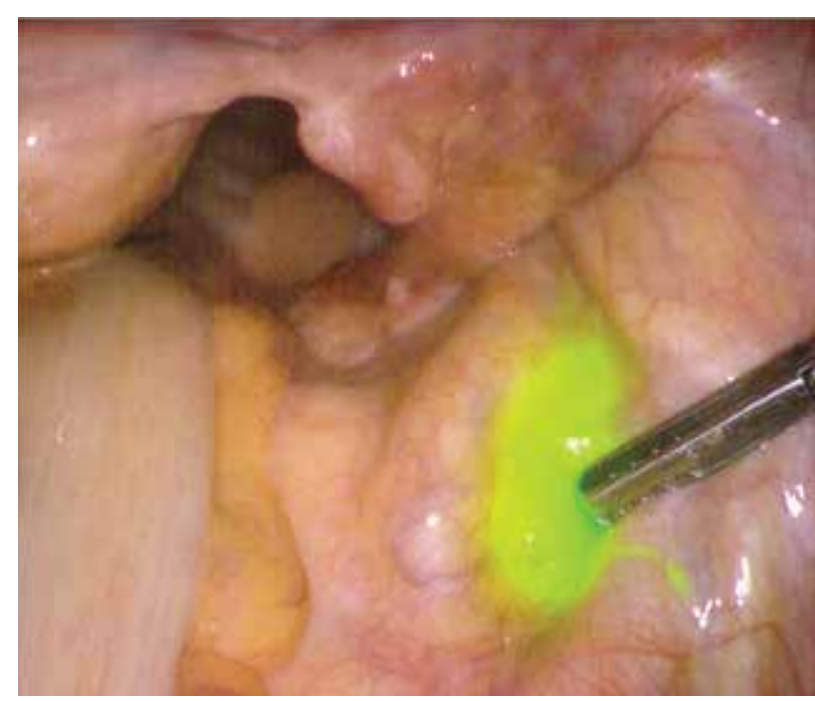

Figure 4 Sentinel lymph node (SLN) mapping after cervical indocyanine green (ICG) injection in endometrial cancer. The right lymphatic trunks and an SLN located between the external iliac and internal iliac artery is shown.

$2.5 \%$ sodium) is injected while the patient is under anesthesia in the operating room. The dye is injected the same way as the radiotracer. A spinal needle or Potocky-type needle is used to inject $4 \mathrm{~mL}$ of dye into the cervical submucosa and stroma. The $4 \mathrm{~mL}$ can be divided into 4 separate injections, 1 into each quadrant of the cervix (1 $\mathrm{mL}$ each). The injections also can be given at the 3 and 9 o'clock positions (Figure 3), which correspond to the parametria and will keep the bladder flap from being stained, which is seen with the

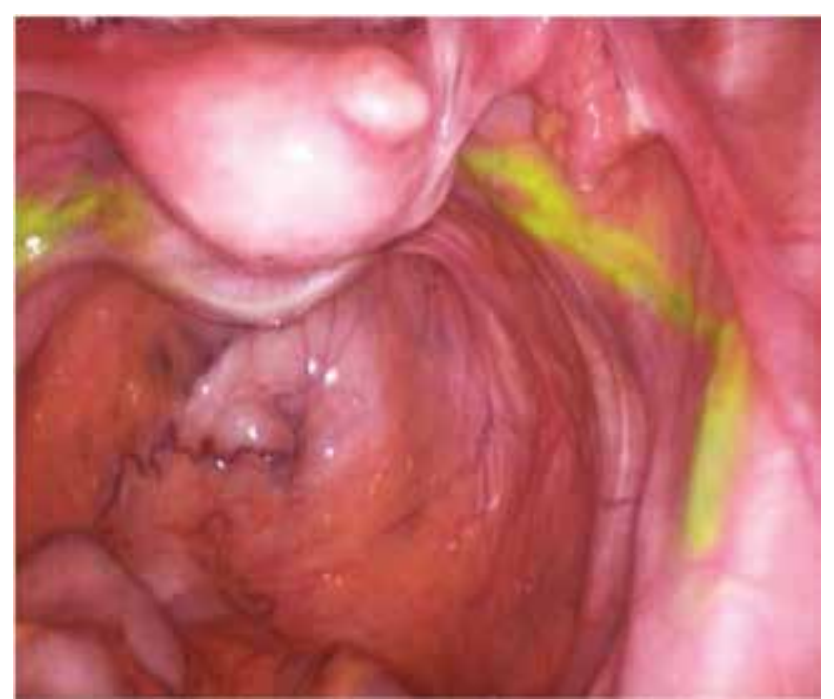

Figure 5 Indocyanine green injected into the cervix, leading into a right external iliac sentinel lymph node. 


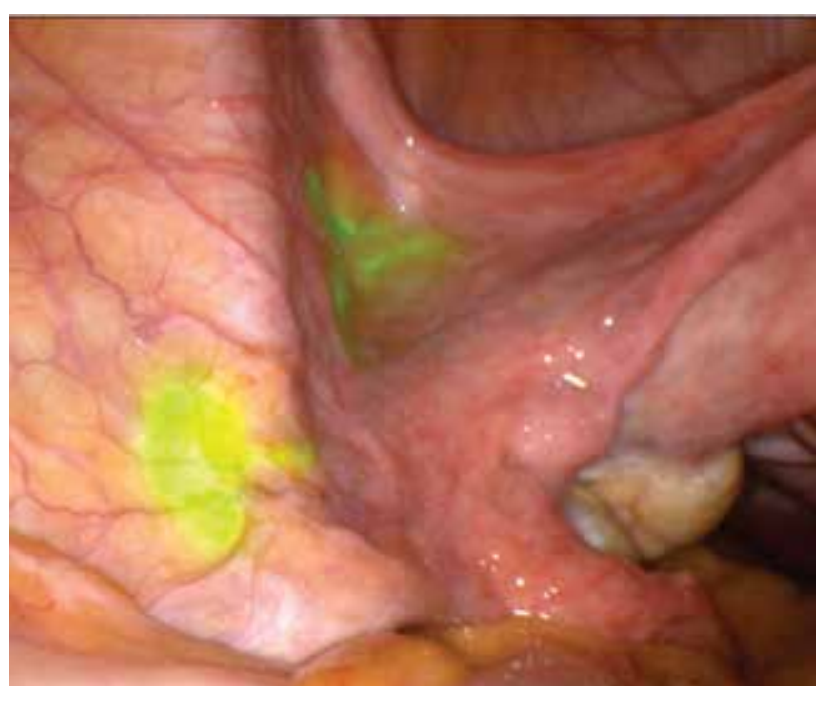

Figure 6 Left pelvic lymphatic trunks with indocyanine green fluorescence imaging after a cervical injection to map endometrial cancer. The sentinel lymph node is located in the medial aspect of the iliac vessels, with a secondary node just lateral to the external iliac artery.

12 o'clock injection. The dye should be injected slowly, at a rate of 5 to 10 seconds per quadrant. ${ }^{25}$

Complications with blue dye are rare, consisting mostly of allergic reactions, including swelling and pruritis of the hands, feet, abdomen, and neck. Severe reactions include edema of the face and glottis, respiratory distress, and shock. Blue dye will also turn the urine blue-green for up to 24 hours.

\section{Fluorescent SLN Imaging With Indocyanine Green}

Indocyanine green (ICG), using near-infrared fluorescence imaging, has recently emerged as an excellent dye for SLN mapping (Figures 4, 5, and 6). The 25- mg dry powder bottle is mixed with $20 \mathrm{~mL}$ of sterile water in the operating room, and 2 to $4 \mathrm{~mL}$ is injected directly into the cervix in similar fashion to that of blue dye. The main contraindication is iodine allergy, which the current US-available product contains. The SLN detection rates with ICG and the bilateral SLN detection rates appear comparable or better than those of blue dye only or radiocolloid. Fluorescent SLN localization with ICG is currently the preferred mapping approach at the authors' institution.

\section{Pathology}

A key component of the added value of SLN procedures in endometrial cancer is enhanced pathology, also known as ultrastaging. At the authors' institution, the protocol for SLN evaluation is described herein. The initial examination is performed using hematoxylin and eosin $(H \& E)$ staining. If the $H \& E$ assessment is negative, 2 adjacent 5 -mcg sections are cut from each paraffin block at each of 2 levels, 50 mcg apart. At each level, one side is stained with $H \& E$ and the other with immunohistochemistry using the anticytokeratin AE1:AE3 (Ventana Medical Systems, Inc., Tucson, AZ) for a total of 4 slides per block. ${ }^{28}$ With immunohistochemistry ultrastaging, an additional $3 \%$ to $4 \%$ of micrometastases to SLNs, which may have been otherwise missed by routine H\&E staining, can be detected. ${ }^{3,29-31}$ Tables 1 and 2 show the results of enhanced pathology and the added value of low-volume metastasis detection.

\section{SLN Mapping Algorithm}

Maintaining a low false-negative rate is a major priority in any SLN program. In a study of 498 patients

\begin{tabular}{|lllll|}
\hline \multicolumn{5}{|c|}{$\begin{array}{l}\text { Table } 1 \\
\text { Incidence of H\&E Macrometastases in Sentinel Lymph Nodes According to Final } \\
\text { Histologic Grade and Depth of Myometrial Invasion }\end{array}$} \\
\hline DMI & Grade 1 & Grade 2 & Grade 3 & Total \\
\hline No invasion & H\&E 0 & H\&E 1 & H\&E 1 & $2 / 242=0.8 \%$ \\
& $\mathrm{n}=165$ & $\mathrm{n}=39$ & $\mathrm{n}=38$ & \\
$<50 \%$ invasion & H\&E 6 & H\&E 4 & H\&E 6 & $16 / 198=8.0 \%$ \\
& $\mathrm{n}=80$ & $\mathrm{n}=62$ & $\mathrm{n}=56$ & \\
$250 \%$ invasion & H\&E 6 & H\&E 3 & H\&E 8 & $17 / 68=25.0 \%$ \\
& $\mathrm{n}=16$ & $\mathrm{n}=15$ & $\mathrm{n}=37$ & $35 / 508=6.9 \%$ \\
\hline
\end{tabular}

Abbreviations: DMI, depth of myometrial invasion; H\&E, hematoxylin and eosin.

From Kim CH, Soslow RA, Park KJ, et al. Pathologic ultrastaging improves micrometastasis detection in sentinel lymph nodes during endometrial cancer staging. Int J Gynecol Cancer 2013;23:967; with permission. 


\begin{tabular}{|c|c|c|c|c|}
\hline DMI & Grade 1 & Grade 2 & Grade 3 & Total \\
\hline No invasion & $\begin{array}{l}\text { MM } 1 \\
\text { ITC } 1 \\
n=165\end{array}$ & $\begin{array}{l}\text { MM } 0 \\
\text { ITC } 0 \\
n=39\end{array}$ & $\begin{array}{l}\text { MM } 0 \\
\text { ITC } 0 \\
n=38\end{array}$ & $2 / 242=0.8 \%$ \\
\hline$<50 \%$ invasion & $\begin{array}{l}\text { MM } 2 \\
\text { ITC 4 } \\
n=80\end{array}$ & $\begin{array}{l}\text { MM } 0 \\
\text { ITC } 4 \\
n=62\end{array}$ & $\begin{array}{l}\text { MM } 0 \\
\text { ITC } 6 \\
n=56\end{array}$ & $16 / 198=8.0 \%$ \\
\hline$\geq 50 \%$ invasion & $\begin{array}{l}\text { MM } 0 \\
\text { ITC } 2 \\
n=16\end{array}$ & $\begin{array}{l}\text { MM } 0 \\
\text { ITC } 0 \\
n=15\end{array}$ & $\begin{array}{l}\text { MM } 1 \\
\text { ITC } 2 \\
n=37\end{array}$ & $5 / 68=7.4 \%$ \\
\hline Total & $10 / 261=3.8 \%$ & $4 / 116=3.4 \%$ & $9 / 131=6.9 \%$ & $23 / 508=4.5 \%$ \\
\hline
\end{tabular}

Abbreviations: DMI, depth of myometrial invasion; ITC, isolated tumor cells; MM, micrometastasis.

From Kim CH, Soslow RA, Park KJ, et al. Pathologic ultrastaging improves micrometastasis detection in sentinel lymph nodes during endometrial cancer staging. Int J Gynecol Cancer 2013;23:968; with permission.

with endometrial cancer who received blue dye cervical injections at Memorial Sloan-Kettering Cancer Center from September 2005 to April 2011, the authors showed that incorporating an SLN mapping algorithm significantly reduced the false-negative rate of the procedure. ${ }^{32}$ An SLN was identified in $401(81 \%)$ of the 498 patients, and correctly diagnosed metastatic nodal disease in 40 of 47 patients who had at least 1 SLN mapped (14.9\% false-negative rate). Applying the algorithm decreased the false-negative rate to $1.9 \%$, because the algorithm takes into account grossly enlarged suspicious nodes and includes a side-specific lymphadenectomy for the nonmapping hemipelvis. Only 1 patient with an isolated positive right para-aortic lymph node was not detected by the algorithm. Sensitivity in- creased from $85.1 \%$ to $98.1 \%$, and the negative predictive value increased from $98.1 \%$ to $99.8 \%$ (Table 3).

The SLN surgical algorithm includes 1) peritoneal and serosal evaluation and washings; 2 ) retroperitoneal evaluation, including the removal of all SLNs and any suspicious nodes; and 3) a side-specific pelvic, common iliac, and interiliac lymph node dissection if there is no mapping on a hemipelvis. A para-aortic lymphadenectomy is left to the attending's discretion (Figure 7). ${ }^{32}$

Since incorporating the SLN mapping algorithm in 2008, the rate of full lymphadenectomy decreased from $65 \%$ to $23 \%$ at the author's institution, with a parallel decrease in median operating room and operative times $(\approx 1$ hour and $\approx 40$ minutes, respectively). The median number

\section{Table 3 Performance of SLN Mapping Alone Compared With the Algorithm for All Patients}

\begin{tabular}{|c|c|c|c|c|c|c|}
\hline & LN Positive & LN Negative & Total & SLN Alone & Calculation & Result \\
\hline SLN positive & 40 & 0 & 40 & Sensitivity & $40 / 47$ & 85.1 \\
\hline \multirow[t]{3}{*}{ SLN negative } & 7 & 354 & 361 & Negative predictive value & $354 / 361$ & 98.1 \\
\hline & 47 & 354 & 401 & False-negative rate & $7 / 47$ & 14.9 \\
\hline & LN Positive & LN Negative & Total & Algorithm & Calculation & Result \\
\hline $\begin{array}{l}\text { Algorithm } \\
\text { positive }\end{array}$ & 53 & 0 & 53 & Sensitivity & $53 / 54$ & 98.1 \\
\hline \multirow{2}{*}{$\begin{array}{l}\text { Algorithm } \\
\text { negative }\end{array}$} & 1 & 420 & 421 & Negative predictive value & $420 / 421$ & 99.8 \\
\hline & 54 & 420 & 474 & False-negative rate & $1 / 54$ & 1.9 \\
\hline
\end{tabular}

Abbreviations: LN, lymph node; SLN, sentinel lymph node.

From Barlin JN, Khoury-Collado $\mathrm{F}$, Kim CH, et al. The importance of applying a sentinel lymph node mapping algorithm in endometrial cancer staging: beyond removal of blue nodes. Gynecol Oncol 2012;125:533; with permission. 
of nodes removed also decreased (from 20 to 7 ). Note, although this article previously reported that 10 or more lymph nodes were required for adequate surgical staging, this benchmark is associated with standard lymphadenectomy and not SLN mapping. The point of SLN mapping is to reduce the number of nodes that need to be removed for staging by targeting those likely to contain metastasis in the hopes of avoiding more extensive dissections and maintaining the ability to find microscopic nodal disease. This decreased rate of comprehensive lymphadenectomy and number of nodes removed has not compromised the rate of detection of metastatic nodal involvement, even in stage IIIC disease. ${ }^{33}$

\section{Moving Forward With SLN Mapping}

The current apprehension with SLN mapping is the associated detection and false-negative rates, a circumstance in which an SLN is found to be negative even though the lymph nodes are found to have metastasis during lymphadenectomy. A failed mapping, on the other hand, is a situation in which an SLN is not identified, which is the opposite of the detection rate, ${ }^{23}$ and in the author's algorithm, a failed mapping requires a sidespecific lymphadenectomy to exclude disease. In the 2009 study of 42 patients with a diagnosis of

\section{Peritoneal and serosal evaluations and washings}

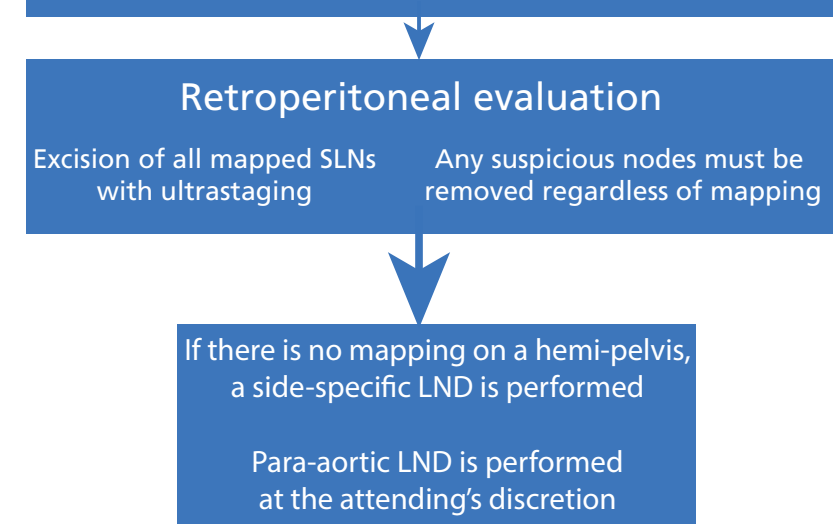

Figure 7 Sentinel lymph node (SLN) mapping algorithm. Abbreviation: LND, lymph node dissection.

From Barlin JN, Khoury-Collado F, Kim CH, et al. The importance of applying a sentinel lymph node mapping algorithm in endometrial cancer staging: beyond removal of blue nodes. Gynecol Oncol 2012;125:534; with permission. grade 1 endometrial carcinoma, all positive cases were detected by the SLN, with no false-negatives. The sensitivity of the SLN procedure in the 36 patients $(86 \%)$ who had an SLN identified was $100 \%$.

An acceptable SLN detection rate varies among practices, but a detection rate of $80 \%$ to $90 \%$ or greater is preferred. ${ }^{26}$ Khoury-Collado et $\mathrm{al}^{26}$ reported on 115 patients with endometrial cancer treated at the author's institution from September 2005 to March 2009 to determine the SLN detection rate for these patients and how many SLN mapping cases a surgeon needed to perform to reach the $90 \%$ benchmark. During the early phase of the study (September 2005-December 2007), an SLN was identified in 50 of 64 cases (78\%), with 2 false-negatives; and during the late phase (January 2008-March 2009), an SLN was identified in 48 of 51 cases (94\%), with no falsenegatives. Detection rates increased from $77 \%$ to $94 \%(P=.033)$ during the 2 periods, with surgeon experience ( $\geq 30$ cases) playing an integral role. ${ }^{26}$

With increasing surgeon experience and a corresponding increase in detection rates of $90 \%$ or greater, combined with a decrease in false-negative rates, SLN mapping can play a more prominent role in lymph node assessment and staging in early-stage endometrial cancer. For now, the standard in many practices continues to include a comprehensive lymphadenectomy versus no nodal assessment, but as its therapeutic benefit comes into question (ASTEC ${ }^{17}$ and the study by Panici et $\mathrm{al}^{18}$ ) and its associated side effects continue to be reported, this may change. However, prospective studies to validate the use of SLN mapping in this patient population are needed and ongoing.

\section{References}

1. Morton DL, Wen DR, Wong JH, et al. Technical details of interoperative lymphatic mapping for early stage melanoma. Arch Surg 1992;127:392-399.

2. Cody HS, Hill AD, Tran KN, Borgen PI. Credentialing for breast lymphatic mapping: how many cases are enough? Ann Surg 1999;229:723-728.

3. Khoury-Collado F, Murray MP, Hensley ML, et al. Sentinel lymph node mapping for endometrial cancer improves the detection of metastatic disease to regional lymph nodes. Gynecol Oncol 2011;122:251-254.

4. Gould EA, Winship T, Philbin PH, Kerr HH. Observations on a "sentinel node" in cancer of the parotid. Cancer 1960;13:77-78. 
5. Cabanas RM. An approach for the treatment of penile carcinoma. Cancer 1977;39:456-466.

6. Burke TW, Levenback C, Tornos $C$, et al. Intraabdominal lymphatic mapping to direct selective pelvic and paraaortic lymphadenectomy in women with high-risk endometrial cancer: results of a pilot study. Gynecol Oncol 1996;62:169-173.

7. Siegel R, Naishadham D, Jemal A. Cancer statistics, 2013. CA Cancer J Clin 2013;63:11-30.

8. Orr JW, Holloway RW, Orr PF, Holimon JL. Surgical staging of uterine cancer: an analysis of perioperative morbidity. Gynecol Oncol 1991;42:209-216.

9. Creasman WT, Odicino F, Maisonneuve P, et al. Carcinoma of the corpus uteri. J Epidemiol Biostat 2001;6:47-86.

10. Zivanovic $O$, Khoury-Collado F, Abu-Rustum NR, Gemignani ML. Sentinel lymph node biopsy in the management of vulvar carcinoma, cervical cancer, and endometrial cancer. Oncologist 2009;14:695-705.

11. Abu-Rustum NR, Khoury-Collado F, Pandit-Taskar N, et al. Sentinel lymph node mapping for grade 1 endometrial cancer: is it the answer to the surgical staging dilemma? Gynecol Oncol 2009;113:163-169.

12. Abu-Rustum NR, Iasonos $A, Z$ hou $Q$, et al. Is there a therapeutic impact to regional lymphadenectomy in the surgical treatment of endometrial carcinoma? Am J Obstet Gynecol 2008;198:e15, discussion e5-6.

13. Barlin JN, Zhou Q, St. Clair CM, et al. Classification and regression tree (CART) analysis of endometrial carcinoma: seeing the forest for the trees. Gynecol Oncol 2013;130:452456.

14. Barakat R, Lev G, Hummer AJ, et al. Twelve-year experience in the management of endometrial cancer: a change in surgical and postoperative radiation approaches. Gynecol Oncol 2007;105:150-156.

15. Partridge EE, Jessup JM, Donaldson ES, et al. 1996 patient care evaluation study (PCE) of cancer of the corpus uteri, National Cancer Database (NCDB), American College of Surgery [abstract]. Gynecol Oncol 1996;72:445. Abstract 10.

16. Abu-Rustum NR, Alektiar $K$, Iasonos $A$, et al. The incidence of symptomatic lower-extremity lymphedema following treatment of uterine corpus malignancies: a 12-year experience at Memorial Sloan-Kettering Cancer Center. Gynecol Oncol 2006;103:714-718.

17. ASTEC study group; Kitchener H, Swart AM, Qian Q, et al. Efficacy of systematic pelvic lymphadenectomy in endometrial cancer (MRC ASTEC trial): a randomized study. Lancet 2009;373:125-136.

18. Panici PB, Basile S, Maneschi F, et al. Systematic pelvic lymphadenectomy vs no lymphadenectomy in early-stage endometrial carcinoma: randomized clinical trial. J Natl Cancer Inst 2008;100:1707-1716.

19. Kilgore LC, Partridge EE, Alvarez RD, et al. Adenocarcinoma of the endometrium: survival comparisons of patients with and without pelvic node sampling. Gynecol Oncol 1995;56:29-33.
20. Todo Y, Kato H, Kaneuchi M, et al. Survival effect of paraaortic lymphadenectomy in endometrial cancer (SEPAL study): a retrospective analysis. Lancet 2010;375:1165-1172.

21. Abu-Rustum NR, Gomez JD, Alektiar KM, et al. The incidence of isolated paraaortic nodal metastasis in surgically staged endometrial cancer patients with negative pelvic lymph nodes. Gynecol Oncol 2009;115:236-238.

22. Hoffman MS, Parsons M, Gunasekaran S, Cavanagh D. Distal external iliac lymph nodes in early cervical cancer. Obstet Gynecol 1999;94:391-394.

23. Abu-Rustum NR, Barakat RR. Observations on the role of circumflex iliac node resection and the etiology of lower extremity lymphedema following pelvic lymphadenectomy for gynecologic malignancy. Gynecol Oncol 2007;106:4-5.

24. Khoury-Collado F, Abu-Rustum NR. Lymphatic mapping in endometrial cancer: a literature review of current techniques and results. Int J Gynecol Cancer 2008;18:1163-1168.

25. Abu-Rustum NR, Khoury-Collado F, Gemignani ML. Techniques of sentinel lymph node identification for early-stage cervical and uterine cancer. Gynecol Oncol 2008;111:S44-50.

26. Khoury-Collado F, Glaser GE, Zivanovic O, et al. Improving sentinel lymph node detection rates in endometrial cancer: how many cases are needed? Gynecol Oncol 2009;115:453-455.

27. Kang S, Yoo HJ, Hwang JH, et al. Sentinel lymph node biopsy in endometrial cancer: meta-analysis of 26 studies. Gynecol Oncol 2011;123:522-527.

28. Pandit-Taskar N, Gemignani ML, Lyall A, et al. Single photon emission computed tomography SPECT-CT improves sentinel node detection and localization in cervical and uterine malignancy. Gynecol Oncol 2010;117:59-64.

29. Kim $\mathrm{CH}$, Soslow RA, Park KJ, et al. Pathologic ultrastaging improves micrometastasis detection in sentinel lymph nodes during endometrial cancer staging. Int J Gynecol Cancer 2013;23:964-970.

30. Kim CH, Barber EL, Khoury-Collado F, et al. Pathologic ultrastaging improves micrometastasis detection in sentinel lymph nodes during endometrial cancer staging [abstract]. Presented at 2013 SGO Annual Meeting on Women's Cancer; March 9-12, 2013; Los Angeles, California. Abstract 176.

31. Kim CH, Khoury-Collado F, Barber EL, et al. Sentinel lymph node mapping: a valuable tool for assessing nodal metastasis in low grade endometrial cancer with superficial myoinvasion [abstract]. Presented at 2013 SGO Annual Meeting on Women's Cancer; March 9-12, 2013; Los Angeles, California. Abstract 22.

32. Barlin JN, Khoury-Collado F, Kim CH, et al. The importance of applying a sentinel lymph node mapping algorithm in endometrial cancer staging: beyond removal of blue nodes. Gynecol Oncol 2012;125:531-535.

33. Leitao MM Jr, Khoury-Collado F, Gardner G, et al. Impact of incorporating an algorithm that utilizes sentinel lymph node mapping during minimally invasive procedures on the detection of stage IIIC endometrial cancer. Gynecol Oncol 2013;129:38- 
SLN Mapping for Endometrial Cancer

\section{Instructions for Completion}

To participate in this journal CE activity: 1) review the learning objectives and author disclosures; 2 ) study the education content; 3 ) take the posttest with a $66 \%$ minimum passing score and complete the evaluation at http://education.nccn.org/ node/40436; and 4) view/print certificate. After reading the article, you should be able to answer the following multiple- choice questions. Credit cannot be obtained for tests completed on paper. You must be a registered user on NCCN.org. If you are not registered on NCCN.org, click on "New Member? Sign up here" link on the left hand side of the Web site to register. Only one answer is correct for each question. Once you successfully answer all posttest questions you will be able to view and/or print your certificate. Software requirements: Internet

\section{Posttest Questions}

1. The standard treatment for early-stage endometrial cancer in many practices includes complete or selective pelvic and para-aortic lymphadenectomy for staging because accurate staging is essential for determining appropriate treatment. a. True

b. False

2. SLN mapping is based on the concept that lymph drains the tumor in the lymphatic system; therefore, if the $\mathrm{SLN}$, or first node, is negative for metastasis, then the ensuing nodes should also be negative.
a. towards
b. away from
c. around

3. Which of the following is/are SLN mapping techniques (based on site of injection)?
a. uterine subserosal
b. cervical
c. endometrial via hysteroscopy
d. all of the above
e. both $b$ and $c$

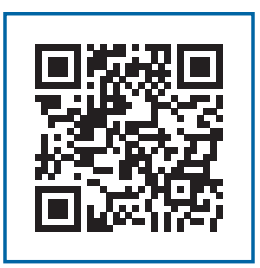

